\title{
Non-Contact Retinal Photocoagulation Using the Volk +90 Dioptre Lens
}

\author{
RICHARD BATES, DAVID WONG and PAUL BLOOR \\ Liverpool
}

\begin{abstract}
Summary
The Volk +90 dioptre lens offers a stereoscopic, wide-field $\left(60^{\circ}\right)$ retinal view without the disadvantage of contact funduscopy. (Fig. 1) Used in conjunction with the slit-lamp, the lens gives a bright, inverted, magnified image of the posterior segment. Its uses in the diagnosis of posterior pole pathology, detailed examination of the optic disc and fundus photography are well established. ${ }^{1-5}$
\end{abstract}

We have used this lens for the application of Argon laser photocoagulation to treat a wide variety of retinal pathology, not only of the posterior pole but as far anteriorly as the equator. It was found that involuntary eye movement was not a significant problem in the light-adapted patient.

The use of this lens offers considerable advantages in that the optics allow access to the peripheral retina with minimal distortion of the fundal view and the laser beam. Fine focussing and adjustment of the laser burn intensity are possible with small movement of the Volk lens. No optical coupling is necessary between the Volk lens and the cornea and this permits the surgeon to utilise both the full range of ocular duction and biomicroscope adjustment to treat anterior retina. Treatment thus involves minimum discomfort for the patient.

We have found this technique particularly useful for:

* patients who are intolerant of contact lenses

* early photocoagulation in patients following cataract extraction

* treatment of peripheral retinal breaks

* patients with media opacities

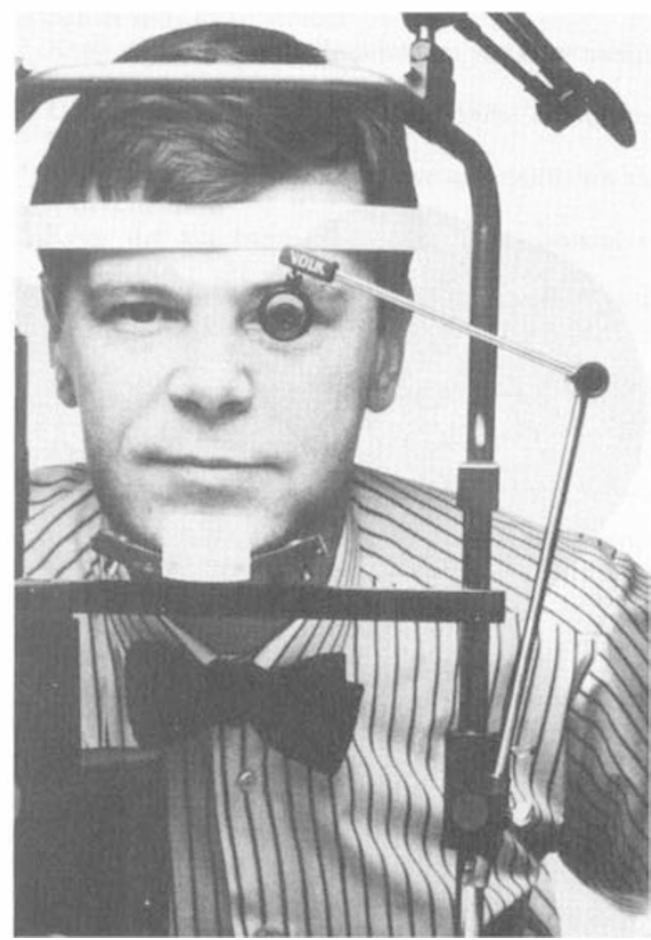

Fig. 1. Volk 90 dioptre lens and Steady Mount. 

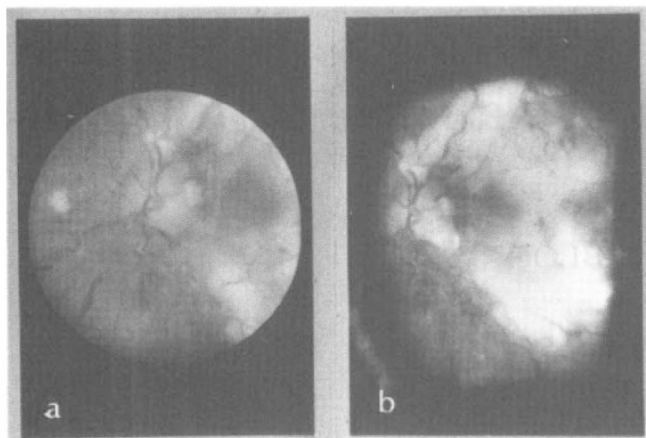

Fig. 2. Posterior pole viewed by: a) $30^{\circ}$ fundus camera, b) the Volk lens and slitlamp.
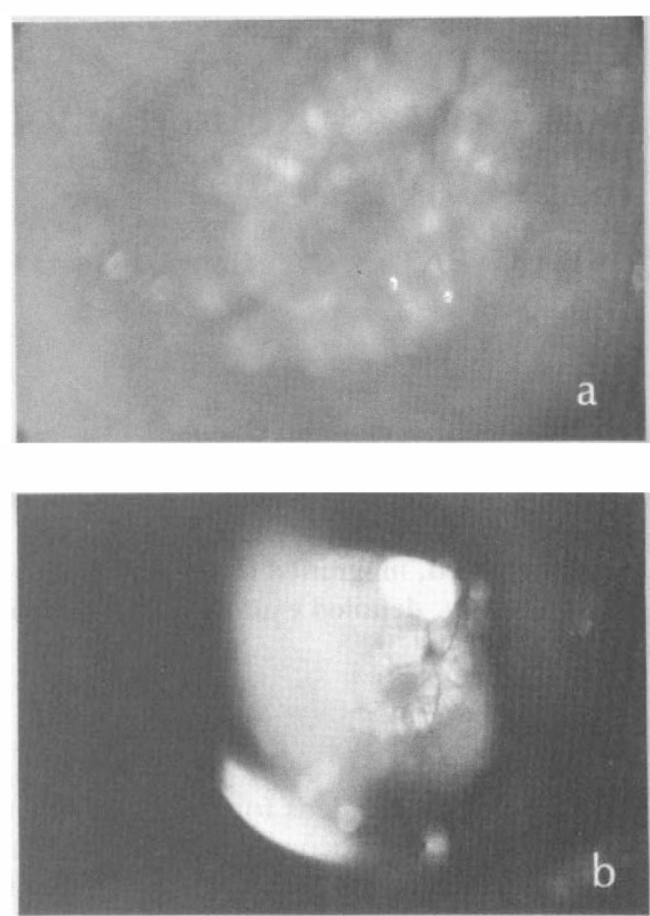

Fig. 3. Peripheral retinal hole after láser retinopexy viewed by: a) $30^{\circ}$ fundus camera, b) the Volk lens.

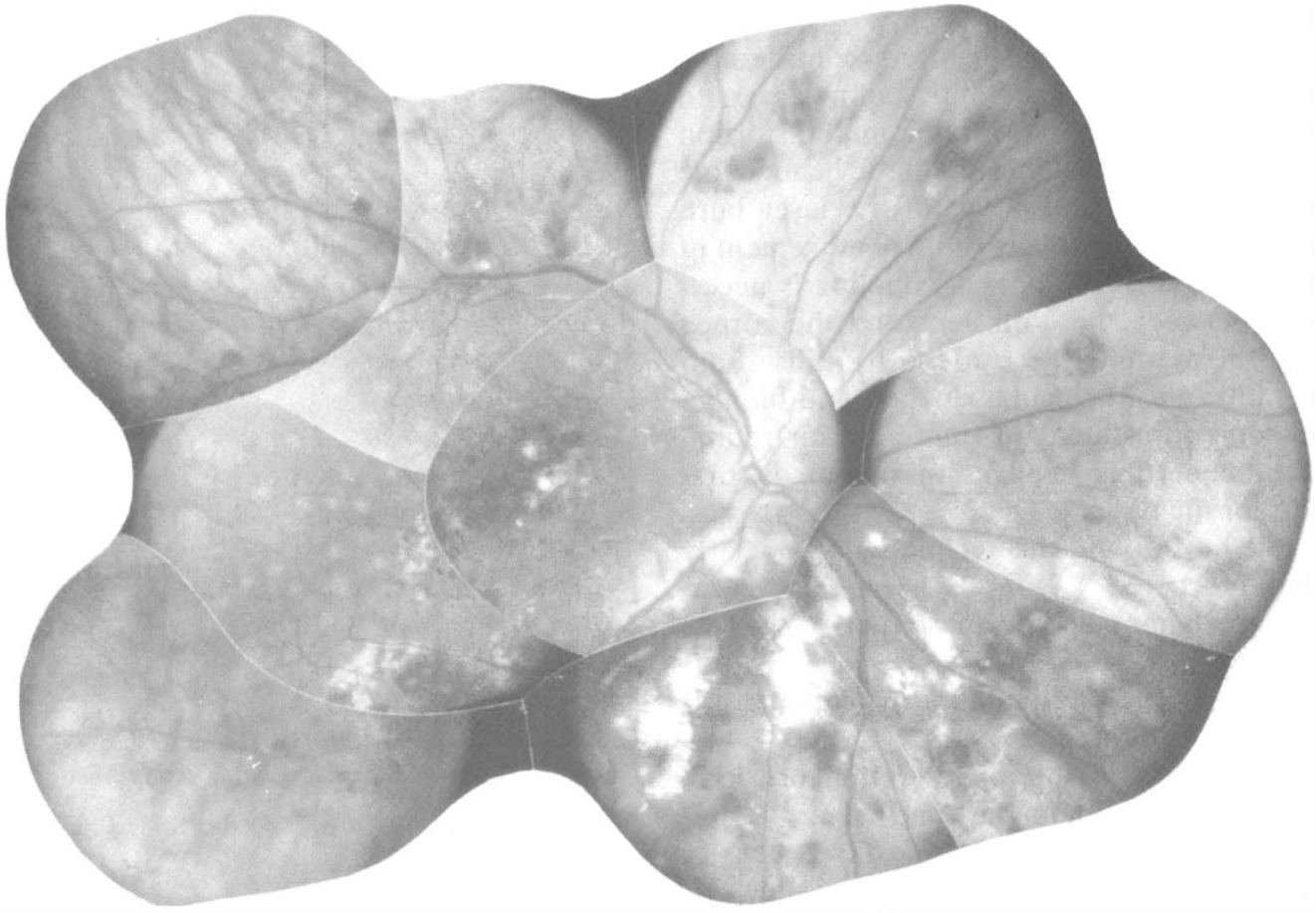

Fig. 4. Panretinal and macular grid laser with Volk lens. 


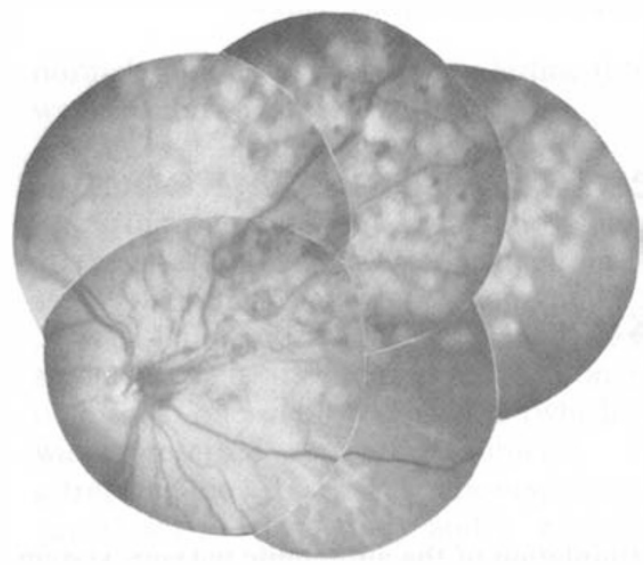

Fig. 5. Sector photocoagulation with Volk lens.

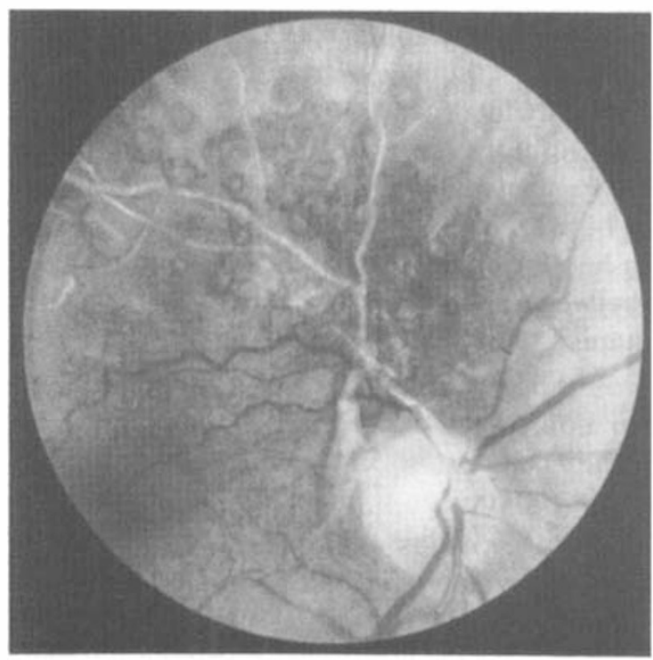

Fig. 6. Retinopexy at posterior pole with Volk lens.

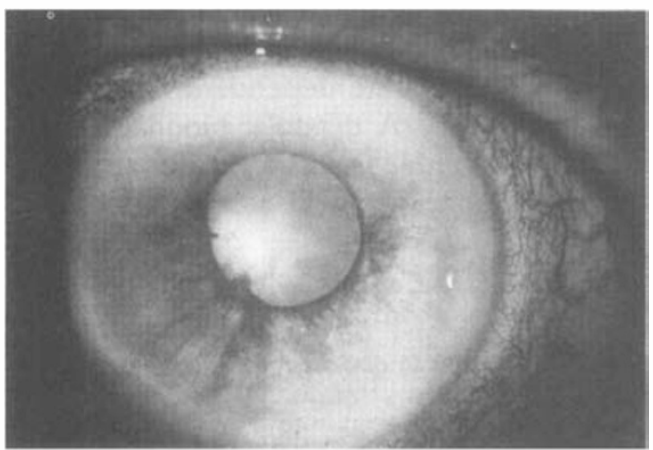

Fig. 7. Media opacity and small pupil - retinal photocoagulation no problem using Volk lens.
Table I

ADVANTAGES

* Non-contact - no pressure on the globe - no infection risk

early post-operative treatment possible

no problems with corneal epithelial dystrophies

* Wide field of view (Fig. 2a, b) easy orientation at the posterior pole easy to change fields - no need to rotate the lens

* Minimal distortion of view and laser in the retinal periphery (Fig. 3)

* Bright image - good stereopsis

* Consistent power delivery (Figs. 4 and 5)

* Image inverted - easy translation from view with 20 or 28 dioptre indirect lens(Fig. 6)

* Through media opacities - view maintained (Fig. 7)

* Patient acceptability - less equipment needed - less mess

\section{Table II}

NOTES ON TECHNIQUE

* Ensure patients' and surgeon's comfort - correct chair height - firm elbow rest

* Steady Mount for lens (Fig. 1)

* Use fixation target for the other eye if feasible

* Light-adapt the eye by using bright illumination before starting treatment

* Keep slit beam width, illumination and laser aiming beam power to a minimum

* Laser settings - are much the same as for the Rodenstock panfunduscope

* Keep the lens as close to the eye as possible for the widest field

* Keep the slit beam and laser beam coaxial to microscope

* Support the patient's upper eyelid with the middle finger, but allow periodic blinks to refresh the tear film

* Clean the lens periodically with an alcoholmoistened cotton wool ball - do not polish it on a white coat!

The authors acknowledge with thanks the assistance of Altomed for providing the Steady Mount, from whom Volk 90 dioptre lenses are available.

\section{References}

${ }^{1}$ El Bayadi G: New Method of Slit Lamp Microophthalmoscopy. Br J Ophthalmol 1953, 37: 625-8.

${ }^{2}$ Rotter H: Technique of Biomicroscopy of the Posterior Eye. Am J Ophthalmol 1956, 42: 409-15.

${ }^{3}$ Rosen E: Biomicroscopic Examination of the Fundus with a +55 Dioptre lens. Am J Ophthalmol 1959, 48: 782-7.

${ }^{4}$ Lundberg C: Biomicroscopic Examination of the Ocular Fundus with a +60 Dioptre Lens. $A m$ J Ophthalmol 1985, 99: 490-1.

5 Whitacre MM: Noncontact Retinal Photocoagulation at the Slit-Lamp Biomicroscope. Am J Ophthalmol 1987, 104: 290-3. 